\title{
Lungenmetastasen beim Mammakarzinom: Resektion doch sinnvoll?
}

\begin{abstract}
Die Leitlinien der Arbeitsgemeinschaft gynäkologische Onkologie (AGO) wie auch der Arbeitsgemeinschaft der Wissenschaftlichen Medizinischen Fachgesellschaften (AMWF) zur Behandlung des metastasierten Mammakarzinoms geben keine eindeutige Empfehlung zur Resektion von Lungenmetastasen. Sie sehen keinen Überlebensvorteil. Damit sind Thoraxchirurgen nicht einverstanden.
\end{abstract}

Die Leitlinien der Arbeitsgemeinschaft gynäkologische Onkologie (AGO) sehen generell keinen Überlebensvorteil einer Resektion eines metastasierten Mammakarzinoms. Allerdings lassen sie es in Einzelfällen bei solitären oder wenigen unilateralen Metastasen oder einem krankheitsfreien Intervall von zwölf Monaten doch offen zu resezieren.

An der generellen Richtigkeit dieser Empfehlungen melden Kölner Arbeitsgruppen unter Leitung von Sascha Macherey nun erhebliche Zweifel an. Er stützt sich dabei auf eine systematische Literaturrecherche in der Medline- und der Cochrane Database of Systematic Reviews für den Zeitraum von 1.1.2000 bis 17.4.2017. Es wurden nur Studien berücksichtigt, die über mindestens 20 Patientinnen mit Metastasektomie, perioperativen Ereignisse und das Langzeitüberleben berichteten. Zudem fand sich noch eine Metaanalyse mit 1.937 Patientinnen mit den einschlägigen Einschlusskriterien. Hier die Resultate:

- Die Lungenmetastasen-Chirurgie erreichte eine postoperative Mortalitätsrate von $0 \%$ bis $3 \%$.

- Das mediane Überleben betrug zwischen 32 und 97 Monaten, die 5-Jahresüberlebenszeiten lagen bei 30 bis $40 \%$.

- Die Resektion von synchronen Lungenmetastasen war nach vorausgegangener Primärtumorresektion mit einem medianen Überleben von bis zu 32 Monaten verbunden.

Schlussfolgerung von Macherey und Mitarbeiter: Die Metastasenchirurgie sollte Patientinnen mit bilateraler Metastasierung oder einem krankheitsfreien Intervall von weniger als zwölf Monate nicht vorenthalten werden. Bei sorgfältiger Indikationsstel-

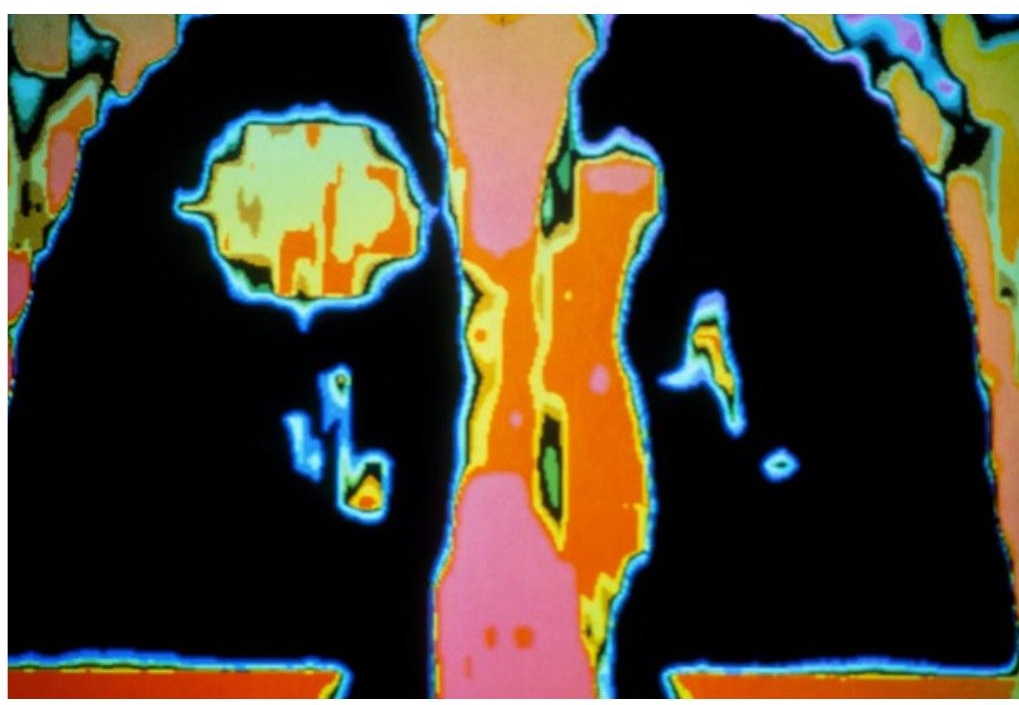

CT eines Mammakarzinoms mit Metastasen in der Lunge. Bisher geht man davon aus, dass die Resektion hier keinen Überlebensvorteil bringt.

lung können auch diese Patientinnen ein günstiges Langzeitüberleben erreichen. Was die Leitlinien betrifft, wäre eine Novellierung durchaus sinnvoll.

Dr. med. Jochen Aumiller

\section{Weitere Infos auf springermedizin.de}

- Optimale Therapie des nichtkleinzelligen Lungenkarzinoms im Stadium IIIB/C

Lungenkrebs gehört trotz der verbesserten Therapieoptionen zu den prognostisch ungünstigen Tumoren.

Mehr zum aktuellen Stand der Therapie des nichtkleinzelligen Lungenkarzinoms im Internet

www.springermedizin.de

- Geben Sie den Titel des Beitrags in die Suche ein.
- 5000 zusätzliche Todesfälle durch Abgas-Mogelei?

Aktuelles zum Dieselskandal: Viele Todesfälle durch Lungenerkrankungen hätten wohl verhindert werden können, wenn die Autobauer sich an die Normen gehalten hätten. 